\title{
GCU
}

Glasgow Caledonian

University

University for the Common Good

\section{Virtually optimized insoles for offloading the diabetic foot: a randomized crossover study}

Telfer, S.; Woodburn, J.; Collier, A.; Cavanagh, P.R.

Published in:

Journal of Biomechanics

DOI:

10.1016/j.jbiomech.2017.06.028

Publication date:

2017

Document Version

Author accepted manuscript

Link to publication in ResearchOnline

Citation for published version (Harvard):

Telfer, S, Woodburn, J, Collier, A \& Cavanagh, PR 2017, 'Virtually optimized insoles for offloading the diabetic foot: a randomized crossover study', Journal of Biomechanics, vol. 60, pp. 157-161.

https://doi.org/10.1016/j.jbiomech.2017.06.028

\section{General rights}

Copyright and moral rights for the publications made accessible in the public portal are retained by the authors and/or other copyright owners and it is a condition of accessing publications that users recognise and abide by the legal requirements associated with these rights.

Take down policy

If you believe that this document breaches copyright please view our takedown policy at https://edshare.gcu.ac.uk/id/eprint/5179 for details of how to contact us. 
1 Title: Virtually optimized insoles for offloading the diabetic foot: a randomized crossover study

2 Running head: Virtually optimized offloading insoles

3 Authors: S Telfer ${ }^{1 *}, \mathrm{~J}_{\text {Woodburn }}^{2}$, A Collier $^{2,3}$, PR Cavanagh $^{1}$

4 1. Department of Orthopaedics and Sports Medicine, University of Washington, WA

5 2. Institute of Applied Health Research, Glasgow Caledonian University, UK

6 3. University Hospital Ayr, UK

7 *Corresponding Author: Department of Orthopaedics and Sports Medicine, UWMC,

8 University of Washington, Box 356500, 1959 NE Pacific St, Seattle, WA 98195, USA. Email:

9 telfers@uw.edu; Tel: +1 2062213964

11 Word count: 3240

12

13 Funding: ST was funded through the People Programme (Marie Skłodowska-Curie Actions) of 14 the European Union's Seventh Framework Programme (FP7 2007-2013) under REA Grant

15 Agreement No. PIOF-GA-2012-329133. The funders had no input into the design, analysis, or 16 decision to publish.

17

18 Conflicts of interest: PC has equity in DiaPEDIA LLC, a manufacturer of custom insoles. ST, 19 AC and JW have no conflicts of interest to declare.

20

21 Clinical trial registration: ISRCTN19805071, www.ISRCTN.org 
2 Integration of objective biomechanical measures of foot function into the design process for

3 insoles has been shown to provide enhanced plantar tissue protection for individuals at-risk of

4 plantar ulceration. The use of virtual simulations utilizing numerical modelling techniques offers

5 a potential approach to further optimize these devices. In a patient population at-risk of foot

6 ulceration, we aimed to compare the pressure offloading performance of insoles that were

7 optimized via numerical simulation techniques against shape-based devices. Twenty participants

8 with diabetes and at-risk feet were enrolled in this study. Three pairs of personalized insoles: one

9 based on shape data and subsequently manufactured via direct milling; and two were based on a

10 design derived from shape, pressure, and ultrasound data which underwent a finite element

11 analysis-based virtual optimization procedure. For the latter set of insole designs, one pair was

12 manufactured via direct milling, and a second pair was manufactured through $3 \mathrm{D}$ printing. The

13 offloading performance of the insoles was analyzed for forefoot regions identified as having

14 elevated plantar pressures. In $88 \%$ of the regions of interest, the use of virtually optimized

15 insoles resulted in lower peak plantar pressures compared to the shape-based devices. Overall,

16 the virtually optimized insoles significantly reduced peak pressures by a mean of $41.3 \mathrm{kPa}(p<$

$170.001,95 \% \mathrm{CI}[31.1,51.5])$ for milled and $40.5 \mathrm{kPa}(p<0.001,95 \% \mathrm{CI}[26.4,54.5])$ for printed

18 devices compared to shape-based insoles. The integration of virtual optimization into the insole

19 design process resulted in improved offloading performance compared to standard, shape-based

20 devices. 
2 For patients with diabetes who have at-risk feet, therapeutic footwear that includes a custom-

3 made insole is recognized as one of the key preventative interventions for reducing plantar

4 ulceration incidence (Bus et al., 2016). It has been demonstrated that for this type of intervention,

5 the inclusion of objective biomechanical measures of foot function within the design process can

6 have a significant protective effect. In a randomized trial, Ulbrecht et al., 2014 found that

7 biomechanically optimized insoles reduced the number of ulcers compared to standard devices,

8 while the number of non-ulcerative lesions remained similar between groups. Bus et al., 2013

9 found a reduction in re-ulceration rates for those with high adherence to wearing optimized

10 footwear when compared to interventions based primarily on shape and subjective clinical

11 assessment, although it should be noted that on an intention to treat basis, no differences were

12 found between groups. These studies utilized plantar pressure distribution measurements, either

13 to derive the shape of the custom insole during the design stage (Ulbrecht et al., 2014), or to

14 provide near real-time feedback on the performance of the therapeutic footwear, allowing an

15 iterative series of pressure relieving modifications to be prescribed during the fitting procedure

16 (Bus et al., 2013). Re-ulceration is multifactorial in nature, however pressure offloading, and

17 thus footwear-plays an important role in maintaining the health of the foot in these patients.

18 Advanced computational simulation methods such as finite element (FE) analysis have provided

19 a number of insights into the biomechanical effects of diabetic foot disease (Telfer et al., 2014a).

20 It has been proposed that these techniques may offer a route to further, objective optimization of

21 therapeutic footwear prior to it being delivered to the patient, both in terms of shape (Chen et al.,

22 2015; Cheung and Zhang, 2005; Spirka et al., 2014) and material properties (Chatzistergos et al.,

23 2015). However, efforts to integrate virtual optimization into the clinical prescription process 
1 have been hampered, both by the complexity of producing these numerical models, and by the

2 time consuming nature of running accurate simulations (Telfer et al., 2014a).

3 We recently reported on efforts to use simplified FE models of the forefoot that were intended to

4 be faster to build and solve in comparison to standard modelling approaches, while still

5 performing to a level of predictive accuracy necessary to provide clinical insights (Spirka et al.,

6 2014; Telfer et al., 2016). This approach utilizes accessible measurement technologies to

7 generate the data required to produce the models and, when applied to insole design, is intended

8 to allow the form and position of pressure relieving features such as metatarsal bars to be

9 optimized for offloading performance.

10 The aim of this study was to test the hypothesis that an insole design workflow incorporating

11 virtual optimization for pressure offloading would provide greater reductions in forefoot plantar

12 pressures in at-risk patients with diabetic foot disease than those produced using the current, 13 shape-based approach.

\section{Methods}

16 Participants

17 Twenty subjects with Type 2 diabetes were enrolled into this study from Ayr University

18 Hospital, UK. Demographic and clinical details are provided in Table 1. Primary inclusion

19 criteria were: peripheral neuropathy, defined as the inability to detect $10 \mathrm{~g}$ monofilament at one

20 or more plantar sites; and elevated barefoot plantar pressures in the forefoot region during gait,

21 defined as peak pressures over $>750 \mathrm{kPa}$ (Owings et al., 2008). The latter criterion was screened 
1 for using an emed pressure platform (Novel GmbH, Munich, Germany), with five trials collected

2 for each foot using a two-step protocol (Bus and de Lange, 2005). Exclusion criteria included

3 Charcot foot and any partial amputation of the foot. Ethical approval for this study was obtained

4 from the local National Health Service committee (reference 14/WS/1150), and participants gave

5 informed, written consent upon enrolment.

7 Protocol

8 An initial assessment was performed during which the data required to produce all sets of custom

9 insoles were collected. During this assessment, physical impressions of both feet were collected using foam boxes. The same researcher performed these impressions for all participants. Then, a

11 series of measurements relating to the internal forefoot anatomy were obtained using ultrasound

12 imaging (MyLab 70; Esaote, Genoa, Italy). In each case, an intraoperative 13MHz linear array

13 probe was used (IOE 323; Esaote, Genoa, Italy). The measurements included tissue thickness

14 under each metatarsal head and sesamoid, the width and sagittal plane radius of the metatarsal

15 heads, and the relative distances between all metatarsal heads and sesamoids.

Dynamic tissue deformation was measured during gait via in-shoe embedded ultrasonography

17 (Telfer et al., 2014b). This technique involves an ultrasound probe being fitted to the sole of the

18 shoe at the interface between the shoe sole and the foot, positioned directly under the $2^{\text {nd }}$

19 metatarsal head. This method allows both the bone and plantar tissue to be imaged, and provides

20 a means of visualizing the dynamic compression of the plantar tissues during gait. The

21 ultrasound system and probe were the same as described previously, and standardized footwear

22 with modifications at the forefoot allowing the probe to be housed and its position adjusted were 
1 provided to the participants. No socks were worn, and a small amount of ultrasound gel was used

2 to maintain contact between the foot and the probe. Using this set up, static ultrasound images

3 were captured with the participant in a relaxed standing position, and cineloops $(47 \mathrm{~Hz})$ showing

4 changes in plantar tissue thickness were recorded while the participant walked at a self-selected

5 speed that was maintained for all walking trials in the study $( \pm 5 \%)$. For the dynamic

6 measurements, data for at least 6 complete gait cycles were collected.

7 Using the same standardized assessment footwear as described previously, in-shoe plantar

8 pressures were measured during relaxed standing and level walking at the same self-selected but

9 controlled speed $( \pm 10 \%)$ using the pedar-X system (Novel GmbH, Munich, Germany). A

10 minimum of 12 steps per foot were recorded at $50 \mathrm{~Hz}$ to ensure reproducibility of the

11 measurements (Arts and Bus, 2011).

Insole design

14 Three pairs of insoles were designed and manufactured for each participant (Fig. 1): 1) standard

15 (shape-based), milled insoles; 2) milled, virtually optimized insoles, and; 3) 3D printed, virtually

16 optimized insoles. The standard insoles were supplied by a single commercial manufacturer with

17 contracts to supply custom insoles to a number of National Health Service clinics attended by

18 people with diabetes and at-risk feet. The supplier was sent the foam box with along with their

19 standard prescription form, which contained clinical information and requested insoles for an at-

20 risk patient with diabetic neuropathy and elevated forefoot pressures, manufactured in EVA with

21 a Shore A hardness of 40 and top cover. The supplier was free to make any modifications to the 
1 design, for example metatarsal pads or bars, as would be their standard practice, (Arts et al.,

2 2015) and followed their standard procedure in the design and manufacture of the devices.

3 For the two virtually optimized pairs of insoles, the foam box foot impressions were 3D scanned

4 (Cubify Sense; 3D Systems, Rock Hill, SC), and this digital object was used as the basis for the

5 virtual simulations and the insole design.

6 The personalized finite element model used to perform the virtual simulations has been described

7 in detail previously (Telfer et al., 2016). Briefly, a computer aided design program (FreeCAD

8 V0.3; www.freecadweb.org) was used to trim the scanned foam box meshes to include just the

9 forefoot surface anatomy. This object was made "watertight" and used to represent the forefoot

10 plantar soft tissues in the model. The ultrasound anatomical measurements were used to generate

11 and position simplified rigid geometric representations of the metatarsals, embedded within the

12 tissue block and inter-metatarsal ligaments used to link these bones. Components representing

13 the floor and shoe were also generated, with geometry and material properties that have been

14 described previously (Telfer et al., 2016).

15 All model components were imported into the finite element analysis software Abaqus, (V6.10;

16 Simulia, Providence, RI). Using the implicit static solver, the in-shoe pressure and displacement

17 data were combined and an iterative process used to determine the material properties of the

18 plantar soft tissue using a second order Ogden hyperelastic model. Material constants were

19 adjusted until predicted contact pressures under the second metatarsal head were within $5 \%$ of

20 those measured experimentally (Petre et al., 2013). Vertical loads were also applied to the other

21 metatarsal heads and modified until they matched the measured pressures under each head at the

22 instance of peak forefoot loading (Spirka et al., 2014). 
1 With the model established, an initial insole CAD design was produced (Rhino V5, McNeel and

2 Associates, WA), based on the shape of the foot scan. This was then imported into the finite

3 element analysis software and added to the existing model (Fig. 2). An iterative series of

4 simulations were run for each foot, where the forefoot geometry of the insole design underwent a

5 standardized modification procedure by increasing the height of a metatarsal bar and removing

6 material under each metatarsal head until the regional peak plantar pressures were predicted to be

$7<200 \mathrm{kPa}$ or the limits of the possible modifications had been reached. The $200 \mathrm{kPa}$ threshold is

8 based on previous studies showing that reducing peak plantar pressures below this level is

9 protective against ulcer recurrence in this patient group (Waaijman et al., 2014).

10 The optimized design was used to make two pairs of insoles, the first milled using the same EVA

11 material as the standard devices, and for the second pair the same designs were 3D printed in a

12 soft PLA material on an Airwolf HD2x printer (Airwolf 3D, Costa Mesa, CA) at a layer

13 resolution of $0.2 \mathrm{~mm}$ and infill of $12 \%$. This second pair was intended to demonstrate the

14 feasibility of producing these devices via 3D printing.

Insole assessment

17 After all pairs of insoles were manufactured, the participant returned for a second experimental session to evaluate them. A randomized crossover design was used to test the effect of each pair

19 of insoles on forefoot offloading, with the participant blinded to the insole type. Walking speed

20 was standardized to within $\pm 10 \%$ of that recorded during the initial assessment session. Footwear

21 was standardized across participants during the insole assessment, with each being provided with

22 extra-depth footwear (Marsden, Peacocks Medical Group Ltd, UK) to wear during testing. As 
1 before, the pedar- $\mathrm{X}$ system recording at $50 \mathrm{~Hz}$ was used to measure the in-shoe plantar pressures,

2 with at least 12 steps being collected for each foot per insole condition. In-shoe pressure

3 measurement insoles were taped to the participants' feet and a thin cotton sock worn over the top

4 to hold the sensor array in place when changing between insole conditions.

5

6 Analysis

7 Data analysis was carried out using R (V3.2.2) (R Development Core Team, 2016). Figures

8 presenting data were produced using the ggplot2 package (Wickham, 2009).

9 Regions of interest were identified from the barefoot plantar pressure data. These regions $\left(1^{\text {st }}\right.$

$10 \mathrm{MTH}, 2^{\text {nd }} \mathrm{MTH}$ and $3-5^{\text {th }} \mathrm{MTHs}$ ) were defined as those where the localized peak plantar

11 pressure, averaged over five steps, exceeded $450 \mathrm{kPa}$ (Owings et al., 2008), and were used for the

12 primary analysis of insole performance. Additionally, to determine how the pressure was

13 redistributed, the midfoot region was also identified and analyzed.

14 For each insole condition, in-shoe pressure measurements for at least 12 steps were analyzed

15 (Arts and Bus, 2011). Regional peak pressures were calculated. Pressure results were compared

16 using a repeated measures ANOVA test followed by appropriate pairwise comparisons.

17 Bonferroni corrections were applied to an initial $\alpha$ of 0.05 where appropriate to correct for

18 multiple comparisons. Peak regional plantar pressures were predicted by the FE models were

19 also compared to the measured values to assess the performance of the modeling approach.

\section{Results}


1 Two participants died due to unrelated medical reasons after enrollment and before it was

2 possible to test their insoles, meaning that eighteen participants completed the study.

3 Seventy-six ROI were identified from the barefoot plantar pressure data. In $88 \%$ of these regions,

4 the milled, optimized design shape showed lower peak pressures than the standard design, with a

5 mean difference of $41.3 \mathrm{kPa}$ (Fig. 3). For the printed optimized insoles, lower peak pressures

6 were seen in $74 \%$ of the regions of interest compared to the standard devices, with a mean

7 difference of 40.5kPa. Repeated measures ANOVA across insole conditions revealed significant

8 differences between groups $(p<0.001)$, with pairwise comparisons showing that both sets of

9 virtually optimized devices provided significantly greater forefoot offloading at regions of

10 interest than the standard insoles (milled: $p<0.001,95 \% \mathrm{CI}[31.1,51.5]$; printed: $p<0.001,95 \%$

11 CI $[26.4,54.5])$. There were no significant differences in offloading performance between the

12 milled and printed optimized insoles.

13 At the midfoot, when compared to the shape based devices, peak pressures were found to

14 increase in the optimized insoles. Peak pressures increased by a mean of $14.1 \mathrm{kPa}(p=0.01,95 \%$

$15 \mathrm{CI}[3.5,24.6])$ and $20.6 \mathrm{kPa}(p<0.00195 \% \mathrm{CI}[11.6,31.5]$ for the milled optimized and the 16 printed optimized designs respectively.

17 The FE models used to design the optimized devices tended to underestimate the measured in-

18 shoe peak plantar pressures by a mean of $35 \mathrm{kPa}$. The mean absolute difference between the

19 predicted and measured results was $68 \mathrm{kPa}(\mathrm{SD} 61)$. 
1 This study describes the implementation of a virtual optimization procedure into the insole

2 design process for diabetic foot offloading and tests these devices in a clinical trial. Despite

3 emerging evidence supporting the inclusion of objective biomechanical measurements within the

4 design process, insoles designed primarily around foot shape - and the inconsistences associated

5 with this (Guldemond et al., 2005) - are still the most common type prescribed for patients with

6 at-risk feet. Here we add to this growing body of evidence, demonstrating that insoles utilizing

7 functional plantar pressure and soft tissue measurements via an FE modeling approach provide

8 improved offloading compared to shape based devices.

9 Our results showed that the primary mechanism used to reduce forefoot pressures in the

10 optimized insoles was a redistribution of loading from the bony prominences of the forefoot to

11 the midfoot. This has been shown to be an effective offloading strategy for custom insoles in

12 number of previous studies (McCormick et al., 2013; Owings et al., 2008). The improvement in

13 offloading effect was similar to that seen in studies testing biomechanically optimized insoles

14 based around pressure and shape (Owings et al., 2008), which meet the offloading performance

15 guidelines recommended by the International Working Group on the Diabetic Foot (S. A. Bus et

16 al., 2016). The present study aimed to test the feasibility of the virtual optimization approach and

17 was based around a comparison against devices used currently in NHS clinical practices, so a

18 direct comparison against the pressure and shape-based insoles was not carried out. Future work

19 will look to evaluate if the addition of the virtual optimization procedures produces benefits beyond that of previous biomechanical optimization approaches.

21 This study focused on elevated forefoot plantar pressure distributions as one of the key

22 mechanisms for plantar ulceration, and as such we are unable to confirm with this study design

23 whether insoles designed using the proposed approach will result in decreased ulceration rates. 
1 Additional biomechanical factors such as shear stresses are likely to play a key role in foot

2 ulceration (Yavuz et al., 2015), and the use of ultrasound gel between the foot and the shoe is

3 likely to affect these. However, given that previous studies on interventions targeting plantar

4 pressures have been shown to be effective in reducing ulcer incidence (Bus et al., 2013; Ulbrecht

5 et al., 2014), we believe these results may act as encouraging early indicators of the insoles

6 potential therapeutic effect. The various activities of daily living may cause different loading

7 patterns on the foot, however in this study we limited the assessment to level, over ground

8 walking.

9 In this study we explored new manufacturing techniques for the manufacture of the insoles,

10 namely 3D printing (also known as additive manufacture). This technology has been the subject

11 of interest for a number of medical applications, with its suitability for mass customization

12 applications making it particularly appealing for the production of therapeutic footwear (Telfer et

13 al., 2012). Our results showed that the milled and printed designs enhanced forefoot offloading

14 by a similar amount, however greater variation in the response was seen for the printed insoles.

15 This may be explained in part by the nature of the printing process used, where the outer layers

16 form a solid shell with a less dense structure making up the interior. By varying the density of

17 the interior structures we attempted to match the material properties of printed devices to the

18 EVA used in the milled devices. However, the solid external shell may lead to areas of increased

19 stiffness around areas with distinct contours, for example at the metatarsal bar feature utilized in

20 these designs, and this, combined with the sensitivity of offloading effect to the positioning of

21 these features (Hastings et al., 2007; Hsi et al., 2005), may have resulted in greater or lesser

22 reductions compared to the softer features on the milled devices. We utilized a relatively mature

23 3D printing technology, fused deposition modeling, which provided functional devices, however 
recent advances in this field present the opportunity to significantly decrease the manufacture time, currently $\sim 6$ hours per insole, and offer the ability to control material properties at a regional level (Tumbleston et al., 2015). These developments have clear implications for the future adoption of this technology in the orthotics field.

In comparison to many previous FE models of the foot, we utilized clinically accessible tools to provide the model inputs, specifically ultrasound, in-shoe pressure measurement and 3D surface scanning. This does increase the time required to collect all of the required data, with a full data collection taking around 60 minutes, and this will have cost implications. The current workflow still requires a level of expertise in modeling, and further work is required to ensure the robustness of the process. The time taken to build and run the optimization process for each insole was approximately two days, the majority of this in running the simulations. This likely remains too long a time period for effective clinical implementation, however there are a number of opportunities within the workflow for further automation. In addition, increased computing power would significantly reduce the modeling time required (Telfer et al., 2016), bringing the time frame down to more feasible levels. With healthcare costs associated with a foot ulcer high (in the US ranging from $\sim \$ 4 \mathrm{~K}$ for a simple case and $\sim \$ 190 \mathrm{~K}$ for more complex case resulting in amputation (Cavanagh et al., 2012), the additional cost of these measurements and the more complex design process could potentially be justified.

The virtual simulations tended to under-predict the measured values. To simplify the modeling procedure as much as possible, we made a number of assumptions when designing the virtual optimization. We looked only at one instance during the gait cycle, that where peak loading of the forefoot occurred. It has been shown that flexion angle can have an effect on material properties (Chen et al., 2014), thus may affect predictions at different points during stance. 
1 Plantar tissue is also viscoelastic and this was not taken into account (Pai and Ledoux, 2011).

2 Future iterations of the model may be adapted to ensure that, for safety, the model over-predicts

3 the pressure values. Nevertheless, the current approach was still found to be effective at

4 enhancing the forefoot offloading performance of the devices.

5

$6 \quad$ Funding

7 ST was funded through the People Programme (Marie Skłodowska-Curie Actions) of the

8 European Union's Seventh Framework Programme (FP7 2007-2013) under REA Grant

9 Agreement No. PIOF-GA-2012-329133. The funders had no role in study design, data collection

10 and analysis, decision to publish, or preparation of the manuscript.

\section{Conflicts of interest}

12 PRC has equity in DiaPEDIA LLC, a manufacturer of custom insoles. 


\section{References}

Arts, M.L.J., Bus, S.A., 2011. Twelve steps per foot are recommended for valid and reliable inshoe plantar pressure data in neuropathic diabetic patients wearing custom made footwear. Clin. Biomech. 26, 880-884.

Arts, M.L.J., de Haart, M., Waaijman, R., Dahmen, R., Berendsen, H., Nollet, F., Bus, S.A., 2015. Data-driven directions for effective footwear provision for the high-risk diabetic foot. Diabet. Med. 32, 790-7.

Bus, S.A., Armstrong, D.G., van Deursen, R.W., Lewis, J.E.A., Caravaggi, C.F., Cavanagh, P.R., International Working Group on the Diabetic Foot, 2016. IWGDF guidance on footwear and offloading interventions to prevent and heal foot ulcers in patients with diabetes. Diabetes. Metab. Res. Rev. 32, 25-36.

Bus, S.A., de Lange, A., 2005. A comparison of the 1-step, 2-step, and 3-step protocols for obtaining barefoot plantar pressure data in the diabetic neuropathic foot. Clin. Biomech. (Bristol, Avon) 20, 892-9.

Bus, S.A., Valk, G.D., van Deursen, R.W., Armstrong, D.G., Caravaggi, C., Hlaváček, P., Bakker, K., Cavanagh, P.R., 2016. The effectiveness of footwear and offloading interventions to prevent and heal foot ulcers and reduce plantar pressure in diabetes: A systematic review. Diabetes. Metab. Res. Rev. 32, 99-118.

Bus, S.A., Waaijman, R., Arts, M., de Haart, M., Busch-Westbroek, T., van Baal, J., Nollet, F., 2013. Effect of Custom-made Footwear on Foot Ulcer Recurrence in Diabetes: A multicenter randomized controlled trial. Diabetes Care 36, 4109-16.

Cavanagh, P., Attinger, C., Abbas, Z., Bal, A., Rojas, N., Xu, Z.-R., 2012. Cost of treating diabetic foot ulcers in five different countries. Diabetes. Metab. Res. Rev. 28 Suppl 1, 10711 .

Chatzistergos, P.E., Naemi, R., Chockalingam, N., 2015. A method for subject-specific modelling and optimisation of the cushioning properties of insole materials used in diabetic footwear. Med. Eng. Phys. 37, 531-8.

Chen, W.-M., Lee, S.-J., Lee, P.V.S., 2015. Plantar pressure relief under the metatarsal heads Therapeutic insole design using three-dimensional finite element model of the foot. J. Biomech. 48, 659-665.

Chen, W.-M., Lee, S.-J., Lee, P.V.S., 2014. The in vivo plantar soft tissue mechanical property under the metatarsal head: implications of tissues' joint-angle dependent response in foot finite element modeling. J. Mech. Behav. Biomed. Mater. 40, 264-74.

Cheung, J.T.-M., Zhang, M., 2005. A 3-dimensional finite element model of the human foot and ankle for insole design. Arch. Phys. Med. Rehabil. 86, 353-8.

Guldemond, N.A., Leffers, P., Schaper, N.C., Sanders, A.P., Nieman, F.H.M., Walenkamp, G.H.I.M., 2005. Comparison of foot orthoses made by podiatrists, pedorthists and orthotists regarding plantar pressure reduction in The Netherlands. BMC Musculoskelet. Disord. 6, 61.

Hastings, M.K., Mueller, M.J., Pilgram, T.K., Lott, D.J., Commean, P.K., Johnson, J.E., 2007. 
Effect of metatarsal pad placement on plantar pressure in people with diabetes mellitus and peripheral neuropathy. Foot ankle Int. 28, 84-8.

Hsi, W.-L., Kang, J.-H., Lee, X.-X., 2005. Optimum position of metatarsal pad in metatarsalgia for pressure relief. Am. J. Phys. Med. Rehabil. 84, 514-20.

McCormick, C.J., Bonanno, D.R., Landorf, K.B., 2013. The effect of customised and sham foot orthoses on plantar pressures. J. Foot Ankle Res. 6, 19.

Owings, T.M., Woerner, J.L., Frampton, J.D., Cavanagh, P.R., Botek, G., 2008. Custom therapeutic insoles based on both foot shape and plantar pressure measurement provide enhanced pressure relief. Diabetes Care 31, 839-44.

Pai, S., Ledoux, W.R., 2011. The quasi-linear viscoelastic properties of diabetic and non-diabetic plantar soft tissue. Ann. Biomed. Eng. 39, 1517-27.

Petre, M., Erdemir, A., Panoskaltsis, V.P., Spirka, T.A., Cavanagh, P.R., 2013. Optimization of nonlinear hyperelastic coefficients for foot tissues using a magnetic resonance imaging deformation experiment. J. Biomech. Eng. 135, 61001-12.

R Development Core Team, 2016. R: A Language and Environment for Statistical Computing.

Spirka, T.A., Erdemir, A., Ewers Spaulding, S., Yamane, A., Telfer, S., Cavanagh, P.R., 2014. Simple finite element models for use in the design of therapeutic footwear. J. Biomech. 47, $2948-55$.

Telfer, S., Erdemir, A., Woodburn, J., Cavanagh, P.R., 2016. Simplified versus geometrically accurate models of forefoot anatomy to predict plantar pressures: A finite element study. J. Biomech. 49, 289-94.

Telfer, S., Erdemir, A., Woodburn, J., Cavanagh, P.R., 2014a. What Has Finite Element Analysis Taught Us about Diabetic Foot Disease and Its Management? A Systematic Review. PLoS One 9, e109994.

Telfer, S., Pallari, J., Munguia, J., Dalgarno, K., McGeough, M., Woodburn, J., 2012. Embracing additive manufacture: implications for foot and ankle orthosis design. BMC Musculoskelet. Disord. 13, 84.

Telfer, S., Woodburn, J., Turner, D.E., 2014b. Measurement of functional heel pad behaviour inshoe during gait using orthotic embedded ultrasonography. Gait Posture 39, 328-32.

Tumbleston, J.R., Shirvanyants, D., Ermoshkin, N., Janusziewicz, R., Johnson, A.R., Kelly, D., Chen, K., Pinschmidt, R., Rolland, J.P., Ermoshkin, A., Samulski, E.T., DeSimone, J.M., Pearce, J.M., Derby, B., Atala, A., Kasper, F.K., Mikos, A.G., Gross, B.C., Erkal, J.L., Lockwood, S.Y., Chen, C., Spence, D.M., Sun, K., Wei, T.S., Ahn, B.Y., Seo, J.Y., Dillon, S.J., Lewis, J.A., Chisholm, G., Kitson, P.J., Kirkaldy, N.D., Bloor, L.G., Cronin, L., Symes, M.D., Kitson, P.J., Yan, J., Richmond, C.J., Cooper, G.J., Bowman, R.W., Vilbrandt, T., Cronin, L., Chakraborty, P., Zuckermann, R.N., Kitson, P.J., Macdonell, A., Tsuda, S., Zang, H.Y., Long, D.-L., Cronin, L., Erkal, J.L., Selimovic, A., Gross, B.C., Lockwood, S.Y., Walton, E.L., McNamara, S., Martin, R.S., Spence, D.M., Zheng, X., Lee, H., Weisgraber, T.H., Shusteff, M., DeOtte, J., Duoss, E.B., Kuntz, J.D., Biener, M.M., Ge, Q., Jackson, J.A., Kucheyev, S.O., Fang, N.X., Spadaccini, C.M., Schaedler, T.A., Jacobsen, A.J., Torrents, A., Sorensen, A.E., Lian, J., Greer, J.R., Valdevit, L., Carter, W.B., Bauer, J., Hengsbach, S., Tesari, I., Schwaiger, R., Kraft, O., Duoss, E.B., Ligon, 
S.C., Husár, B., Wutzel, H., Holman, R., Liska, R., Yagci, Y., Jockusch, S., Turro, N.J., Dendukuri, D., Panda, P., Haghgooie, R., Kim, J.M., Hatton, T.A., Doyle, P.S., Dendukuri, D., Pregibon, D.C., Collins, J., Hatton, T.A., Doyle, P.S., Gonzalez-Meijome, J.M., Compañ-Moreno, V., Riande, E., Rogers, J.A., Someya, T., Huang, Y., Bauer, S., BauerGogonea, S., Graz, I., Kaltenbrunner, M., Keplinger, C., Schwödiauer, R., Travitzky, N., Bonet, A., Dermeik, B., Fey, T., Filbert-Demut, I., Schlier, L., Schlordt, T., Greil, P., Cvetkovic, C., Raman, R., Chan, V., Williams, B.J., Tolish, M., Bajaj, P., Sakar, M.S., Asada, H.H., Saif, M.T., Bashir, R., Lu, Y., Mapili, G., Suhali, G., Chen, S., Roy, K., Suh, S.K., Bong, K.W., Hatton, T.A., Doyle, P.S., 2015. Additive manufacturing. Continuous liquid interface production of 3D objects. Science 347, 1349-52.

Ulbrecht, J.S., Hurley, T., Mauger, D.T., Cavanagh, P.R., 2014. Prevention of Recurrent Foot Ulcers With Plantar Pressure-Based In-Shoe Orthoses: The CareFUL Prevention Multicenter Randomized Controlled Trial. Diabetes Care 37, 1982-9.

Waaijman, R., de Haart, M., Arts, M.L.J., Wever, D., Verlouw, A.J.W.E., Nollet, F., Bus, S.A., 2014. Risk factors for plantar foot ulcer recurrence in neuropathic diabetic patients.

Diabetes Care 37, 1697-705.

Wickham, H., 2009. ggplot2: elegant graphics for data analysis. Springer New York. Springer, New York.

Yavuz, M., Master, H., Garrett, A., Lavery, L.A., Adams, L.S., 2015. Peak Plantar Shear and Pressure and Foot Ulcer Locations: A Call to Revisit Ulceration Pathomechanics. Diabetes Care 38, e184-5. 
1 Tables

2 Table 1 - Participant information

\begin{tabular}{ll}
\hline Variable & $5 / 15$ \\
\hline Sex (F/M) & $64.4(\mathrm{SD} 9.2)$ \\
Age (years) & $87.8(\mathrm{SD} 16.1)$ \\
Weight $(\mathrm{kg})$ & $1.73(\mathrm{SD} 0.09)$ \\
Height $(\mathrm{m})$ & $17.5(\mathrm{SD} 12.3)$ \\
Diabetes duration (years) & $5 / 15$ \\
Ulcer history (Y/N)
\end{tabular}

3

4 


\section{Figure legends}

2 Fig. 1 - Exemplar insole designs: A) standard shape based, direct milled; B) virtually optimized,

3 direct milled; C) virtually optimized, 3D printed

$4 \quad$ Fig. 2 - FE model with construction overview

5 Fig. 3 - Peak pressure results for each insole condition are presented as boxplots. The box

6 signifies the upper and lower quartiles, and the mean is represented by the black line within the

7 box. Statistically significant differences between conditions are indicated with “*” 
$1 \quad$ Figure 1

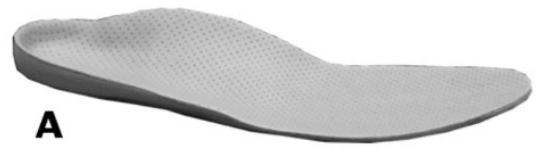

B
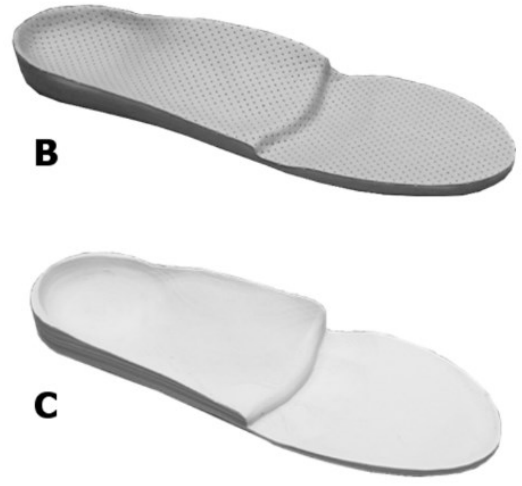

2

3 
$1 \quad$ Figure 2

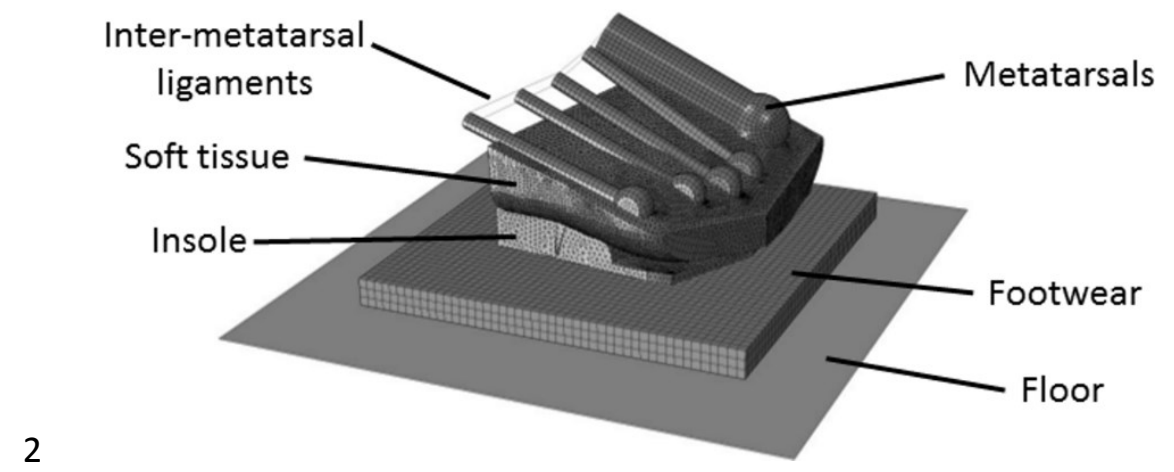


$1 \quad$ Figure 3

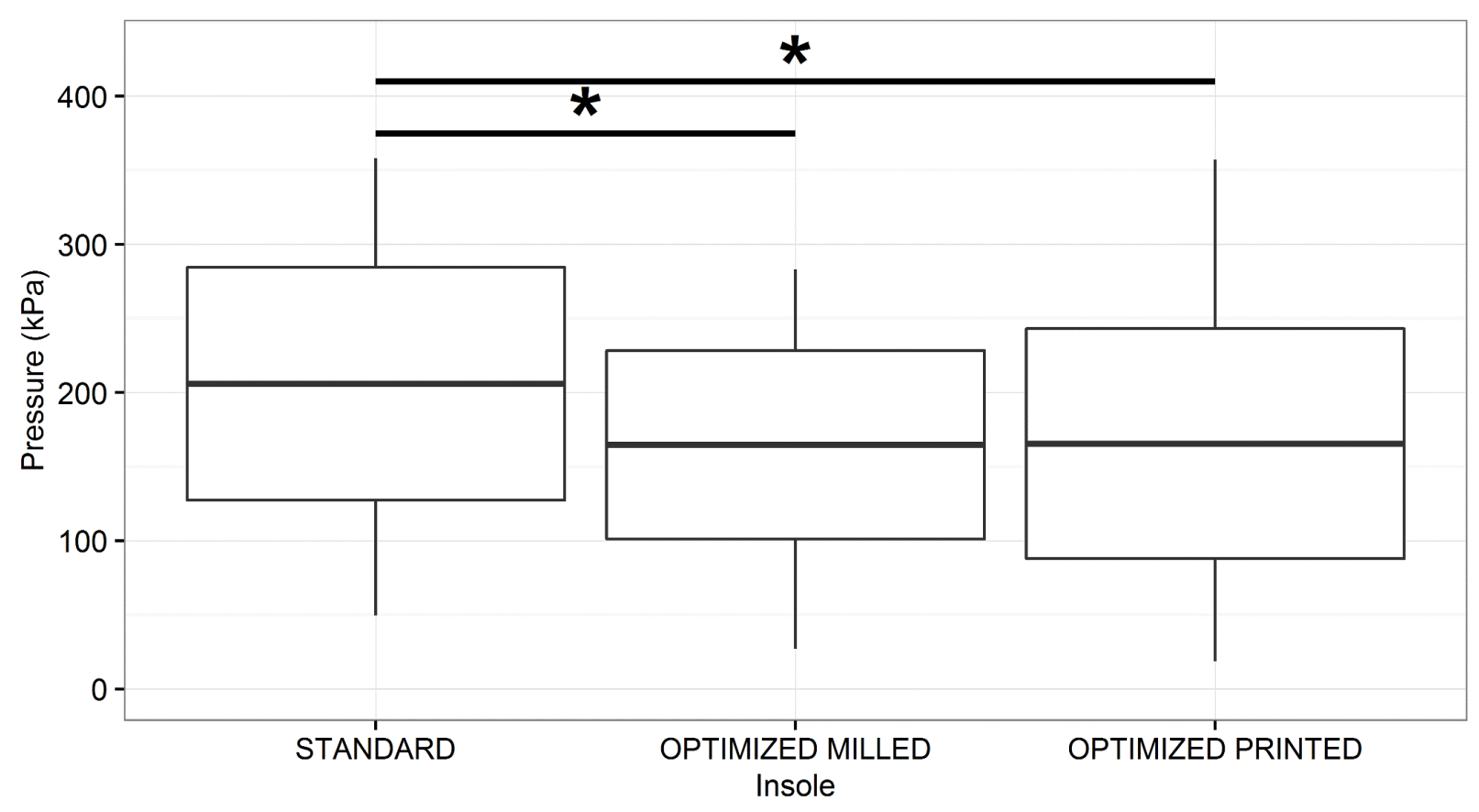

2 